\title{
Aplicación del método de inversión usando mínimos cuadrados (dls) para el procesamiento de sondeos eléctricos verticales en Campo Escuela Colorado (Santander) - caso de estudio
}

\section{Application of the least squares inversion method for vertical electrical sounding processing in the Colorado field (Santander) - a case study}

\author{
Y.P Goyes Peñafiel ${ }^{1}$, G Jiménez Diaz ${ }^{2}$, R Mier Umaña ${ }^{3}$ \\ ${ }^{1}$ Geólogo, MSc Candidato Facultad de Geología, Universidad Estatal de Perm (Rusia), Krai de Perm, Perm, Federación de Rusia, \\ goyes.yesid@gmail.com \\ ${ }^{2}$ GIGBA, Geólogo PhD, Universidad Industrial de Santander, Bucaramanga, Colombia, gjimenez@uis.edu.co \\ ${ }^{3}$ Geólogo, Universidad Industrial de Santander, Bucaramanga, Colombia, rimier@uis.edu.co
}

Recibido: abril 14, 2016. Aceptado: may 27, 2016. Versión final: jun 01, 2016

\begin{abstract}
RESUMEN
En el método de resistividad eléctrica se inducen corrientes eléctricas en el subsuelo y se mide la diferencia de potencial en la superficie. En un subsuelo con capas de diferentes características físicas, las medidas de resistividad y sus respectivas anomalías contribuyen con la prospección y caracterización de las propiedades eléctricas de los diferentes estratos. La resistividad es una de las propiedades físicas más variables, ya que se puede ver afectada por pequeños cambios del clima o irregularidades del subsuelo, para ciertos tipos de materiales, el agua puede modificar sus propiedades eléctricas; en suelos saturados o con niveles freáticos, la resistividad de un material tiende a disminuir. La distribución de los rangos de resistividad más bajos se analiza para diferentes puntos con el fin de estimar una distribución superficial de estos niveles saturados. En Campo Escuela Colorado se realizó un levantamiento de 8 sondeos eléctricos verticales, el procesamiento e interpretación de los datos adquiridos se realizó por medio del desarrollo de una inversión usando mínimos cuadrados para estimar la resistividad real y profundidades que mejor se ajusten a un modelamiento de la curva teórica.
\end{abstract}

Palabras Clave: Método eléctrico, Campo Escuela Colorado, sondeo eléctrico vertical, nivel freático, acuífero, inversión.

\begin{abstract}
In the resistivity method, artificially generated electric currents are introduced into the ground and the resulting potential differences are measured at the surface, in subsurface layers of different physical characteristics, resistivity measurements and their respective anomalies provide information on the form and electrical properties of subsurface inhomogeneities. Resistivity is one of the most variable physical properties because it can be affected by small changes in climate or subfloor, for certain types of materials, water can modify their electrical properties; in saturated soil or water tables, the resistivity of a material tends to decrease. The distribution of lower resistivity ranges for different points are analyzed in order to estimate a surface distribution of these saturated levels. At Colorado Field was survey eight vertical electrical sounding; processing and interpretation of acquired data is done through the development of an investment using least squares to estimate the actual resistivity and depths that are best suited to modeling the theoretical curve.
\end{abstract}

Key words: Electrical method, Colorado Field, vertical electrical sounding, water table, aquifer, inversion.

\section{INTRODUCCIÓN}

El Campo Escuela Colorado se localiza en la Cuenca Valle del Medio del Magdalena (VMM), en la Provincia Estructural del Piedemonte Occidental de la Cordillera Oriental; al sureste de la ciudad de Barrancabermeja y al sur del Campo La Cira-Infantas (Figura 1). Estratigráficamente en el Campo Escuela Colorado se encuentra sobre la Formación Colorado (Gómez et al., 2008 y Zafra, 2013) compuesta por intercalaciones de paquetes de areniscas de grano fino a medio y lodolitas rojizas con meteorización esferoidal, las areniscas

Este artículo puede compartirse bajo la licencia CC BY-ND 4.0 (https://creativecommons.org/licenses/by-nd/4.0/)Y.P Goyes Peñafiel, G Jiménez Diaz, R Mier Umaña. "Aplicación del método de inversión usando mínimos cuadrados (dls) para el procesamiento de sondeos eléctricos verticales en campo escuela colorado (Santander) - caso de estudio”, UIS Ingenierías, vol. 15, no. 2, pp. 125-133, jul-dic 2016,doi:10.18273/revuin.v15n2$\underline{2016011 .}$ 
presentan estratificación cruzada, paralela y sub-paralela, con espesores que varían entre 30 a $70 \mathrm{~cm}$ y las lodolitas rojizas entre 10 a $20 \mathrm{~cm}$. según Caballero et al. (2010). Su contacto inferior es una discordancia erosiva y es reconocible por un nivel areniscas marrones granulosos con cemento calcáreo y nódulos de manganeso y encima de estas un nivel de paleosuelo con costras calcáreas sobre arenitas y lodolitas moteadas de la Formación Mugrosa. En el flanco Este del Sinclinal de Nuevo Mundo, la Formación Colorado alcanza un espesor de 1400 m y se evidencia un grupo de 3 ciclos principalmente granocrecientes y estratocrecientes los cuales hacia el Norte presentan facies proximales y medias correspondientes a un abanico aluvial.

La zona de trabajo presenta una influencia hídrica asociada principalmente al Caño El Canelo - Río la Colorada - Río Fuego (Figura 1B). Estos drenajes se encuentran controlados por las Fallas La Colorada y Cascajales, ambas con componente de rumbo sinestral (Zafra, 2013). La presencia de fallas, litología permeable e impermeable con fracturas internas y las condiciones hidrológicas permiten sugerir que la zona es apta para la exploración de aguas subterráneas como fuente de agua potable para la comunidad residente la zona.
El objetivo principal de este trabajo es evaluar las características hidrogeológicas del Campo Escuela Colorado utilizando el método de Sondeos Eléctricos Verticales (SEV), estimando así las propiedades resistivas del subsuelo y su distribución superficial y determinar potenciales acuíferos que puedan beneficiar directamente a la comunidad. La exploración se realizó con $8 \mathrm{SEVs}$ distribuidos sobre las zonas más cercanas a las fuentes hídricas principales, en la Figura 2 se muestra el error porcentual para cada SEV; el error es menor de $8 \%$ para todas las medidas. Los datos son procesados usando un algoritmo que resuelve un problema básico de mínimos cuadrados como parte de la inversión de datos de resistividad.

\section{1 MARCO TEÓRICO}

Para aplicaciones de ingeniería, los ensayos geofísicos son usados para caracterizar el subsuelo más somero a partir de la medición de alguna propiedad física de los materiales que lo componen (resistividad, velocidad, gravedad, magnetismo, etc.). El subsuelo somero está generalmente considerado para profundidades menores a unos centenares de pies (Butler, 2008).

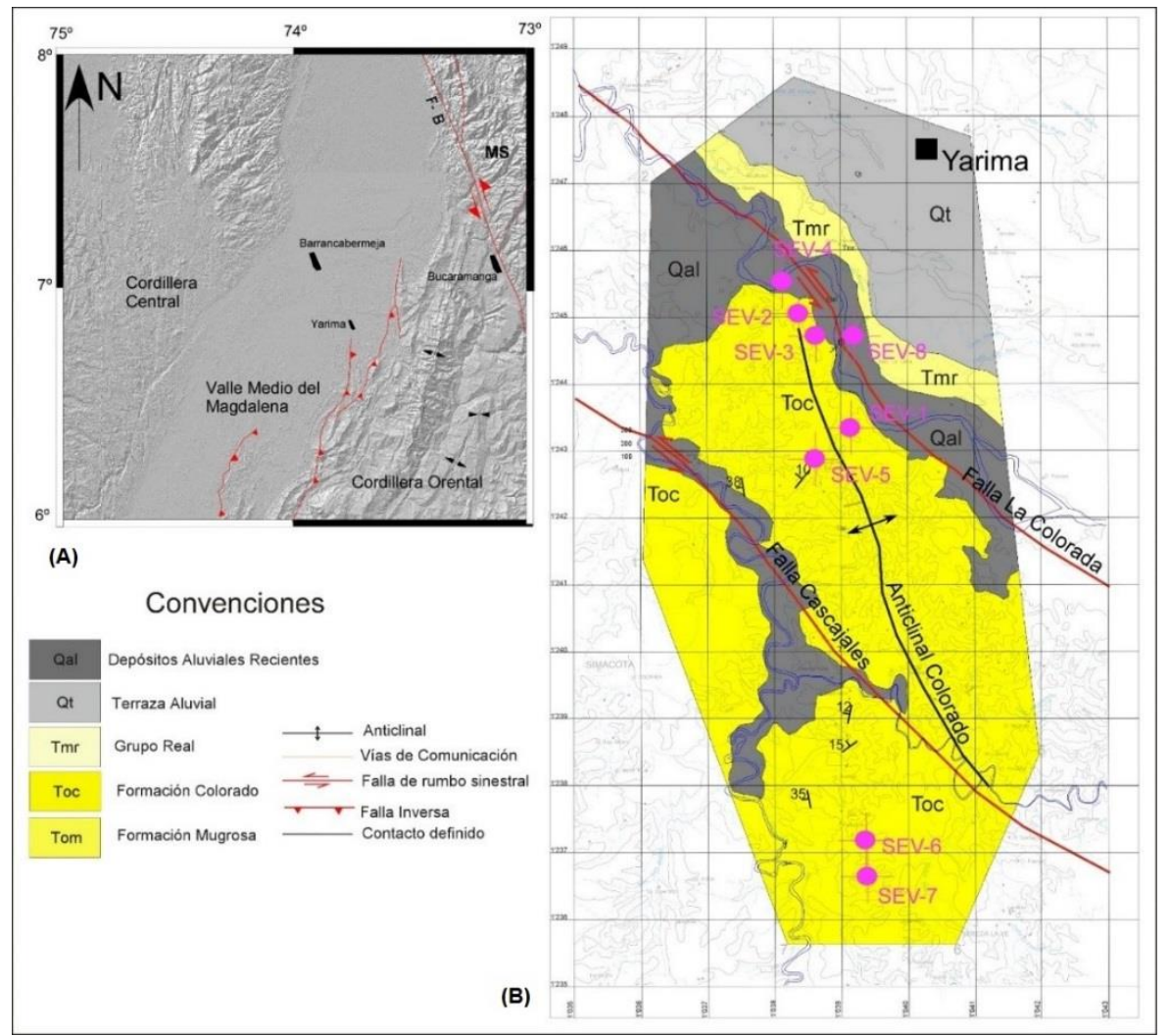

Figura 1. (A) Mapa regional de localización del área de trabajo, En la zona de Yarima. (B) Geología del Campo Colorado y los sondeos eléctricos verticales distribuidos sobre las zonas de influencia de los drenajes principales 


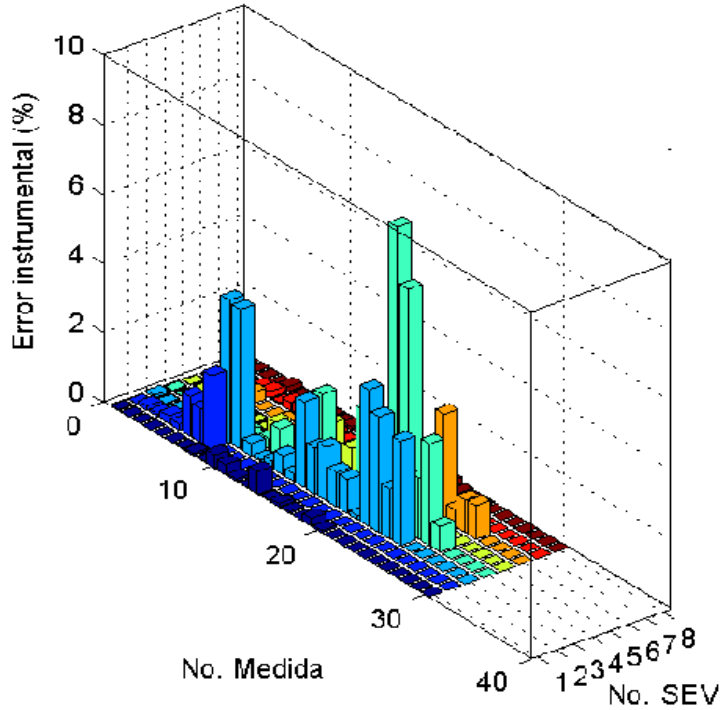

Figura 2. Distribución porcentual de la medida en cada Sondeo eléctrico Vertical (SEV) adquirido en Campo Escuela Colorado. En la gráfica se muestra un máximo porcentual de $8 \%$ para el SEV-5, los porcentuales para los demás SEV no superan el $4 \%$.

La exploración geofísica se aplica para estudios de litología, evaluación de fallas, delimitación del basamento rocoso, determinación de aguas subterráneas y espesores de suelos transportados o residuales, entre otros (Steeples, 2001).

El principio teórico para el método de resistividad eléctrica se basa en la ley de ohm según la ecuación (1), la cual estudia las caídas de potencial de una corriente que atraviesa un material de cierta longitud y resistividad característica. Para este experimento la resistividad depende de la resistencia del material y de la geometría cilíndrica (ecuación 1.2).

$$
\begin{gathered}
V=I * R \\
V_{c}=\rho \frac{I}{2 \pi}\left(\frac{1}{r_{A}}-\frac{1}{r_{B}}\right) \\
\Delta V=\frac{\rho I}{2 \pi r}\left\{\left(\frac{1}{r_{A}}-\frac{1}{r_{B}}\right)-\left(\frac{1}{R_{A}}-\frac{1}{R_{B}}\right)\right\}
\end{gathered}
$$

Considerando la resistencia $R$ como la propiedad de un material homogéneo e isótropo se reemplaza los términos de la ecuación (1.2) en la ecuación (1.1), obteniendo así la ecuación de resistividad eléctrica. Considerando los términos de la ecuación (1.3), para la adquisición de datos de resistividad eléctrica $(\rho)$ se deben tomar varias mediciones para diferencias de potencial $(V)$ y corriente ( $I$ ) a diferentes distancias $r_{i}$ (Figura 3).

En un medio homogéneo, la medición de la resistividad será equivalente al valor de resistividad verdadero en un punto dado de dicho medio. Sin embargo, la ocurrencia de un medio de esas condiciones es muy rara y prácticamente no existe en el subsuelo real. Esencialmente la resistencia aparente es un valor promedio registrado por una masa de suelo energizado durante una medida. Numéricamente la resistencia aparente puede ser expresada por la ecuación (1.4)

$$
\rho_{a}=\frac{\Delta V}{I} G
$$

El coeficiente geométrico ( $G$ ) varía según el tipo de arreglo para cada medición. El espaciamiento y la distribución de los electrodos de potencial inducen cierto campo equipotencial que excita las partículas de suelo. Estos tipos de arreglo son conocidos como Wenner, Schlumberger y dipolo-dipolo.

\section{METODOLOGÍA}

La adquisición de datos de campo se realizó mediante el tipo de arreglo schlumberger, el cual es muy utilizado en zonas con poca variabilidad lateral en los datos de resistividad real (Hubbard, 2009).

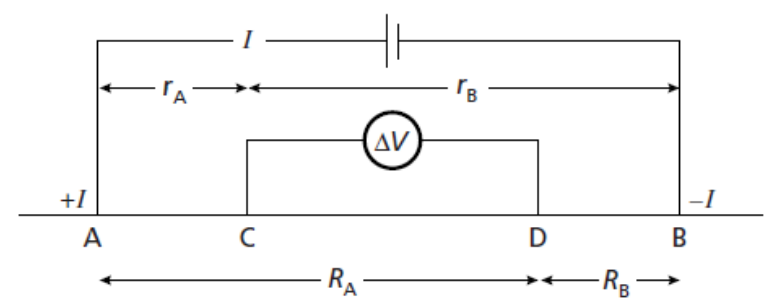

Figura 3. Esquema generalizado para la configuración de los electrodos en mediciones para resistividad eléctrica. Modificado de (Hubbard, 2009).

En este tipo de arreglo los electrodos de potencial deben estar separados una distancia máxima que no supere 5 veces la distancia $(L<5 l)$ entre los electrodos de corriente como se muestra en la Figura 4 y su respetiva expresión física en las ecuaciones (2.1) y (2.2). El arreglo tipo schlumberger es de mayor preferencia para los SEVs. La principal limitación se refiere a términos geométricos debido a las grandes distancias que se deben abarcar para aumentar la exploración en profundidad. En la Tabla 1 se muestra las coordenadas en el sistema Colombia_Bogota_Zone de todos los SEVs con su apertura máxima ( $L$ ), la profundidad final del modelo resistividad - profundidad depende directamente de la apertura en una relación aproximada de $\mathrm{h}=0.25 *(L)$; por lo tanto, para este levantamiento de datos de resistividad se espera un rango de profundidades desde $5 \mathrm{~m}$ hasta 35 $\mathrm{m}$. 
TABLA I. LOCALIZACIÓN DE SONDEOS ELÉCTRICOS VERTICALES Y APERTURA MÁXIMA (AB/2).

\begin{tabular}{|c|c|c|c|c|}
\hline SONDEO & ESTE $(\mathrm{m})$ & NORTE $(\mathrm{m})$ & ALTURA $(\mathrm{msnm})$ & Apertura máxima $[\mathrm{L}](\mathrm{m})$ \\
\hline SEV-1 & 1039132 & 1243355 & 141 & 150 \\
\hline SEV-2 & 1038365 & 1345050 & 110 & 30 \\
\hline SEV-3 & 1038611 & 1244731 & 133 & 400 \\
\hline SEV-4 & 1038127 & 1245514 & 113 & 350 \\
\hline SEV-5 & 1038610 & 1242896 & 150 & 215 \\
\hline SEV-6 & 1039342 & 1237228 & 155 & 300 \\
\hline SEV-7 & 1039388 & 1236684 & 155 & 125 \\
\hline SEV-8 & 1039182 & 1244709 & 119 & 200 \\
\hline
\end{tabular}

$$
\rho_{a}=\frac{\pi}{2 l} \frac{\left(L^{2}-x^{2}\right)^{2}}{\left(L^{2}+x^{2}\right)} \frac{\Delta V}{I}
$$

Si todas las medidas tienen simetría ( $x=0$ ) entonces,

$$
\rho_{a}=\frac{\pi L^{2}}{2 l} \frac{\Delta V}{I}
$$
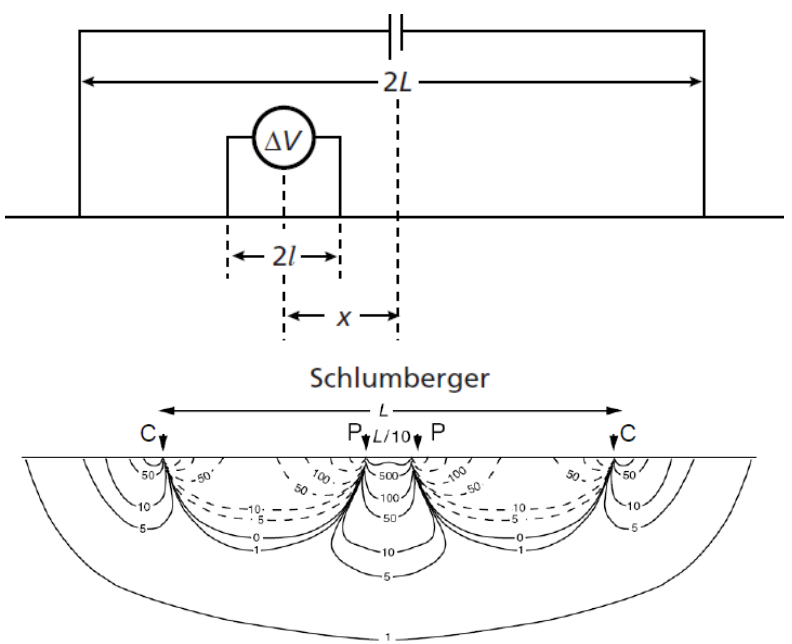

Figura 4. Arreglo tipo Schlumberger para sondeos eléctricos verticales. Modificado de Kearey et al. (2002)

Para el procesamiento de los SEVs se usa un algoritmo que optimiza el ajuste entre los datos observados y los teóricos de resistividad aparente según lo descrito por Ekinci \& Demirci (2008). La expresión matemática (ecuación 2.3) para el modelamiento del arreglo tipo schlumberger en un medio isótropo y homogéneo de resistividad aparente $\left(\rho_{a}\right)$ es descrita por Koefoed (1970).

$$
\rho_{a}=s^{2} \int_{0}^{\infty} T(\lambda) J_{1}(\lambda s) \lambda \partial \lambda
$$

Donde, $s$ es el espaciamiento entre electrodos $(L), J_{1}$ denota la función de Bessel de primer orden y $\lambda$ es una variable de integración. La función transformada de resistividad, $T(\lambda)$, es dada por la siguiente relación:

$$
T_{i}(\lambda)=\frac{T_{i+1}(\lambda)+\rho_{i} \tanh \left(\lambda h_{i}\right)}{1+T_{i+1}(\lambda) \tanh \left(\lambda h_{i}\right) / \rho_{i}}
$$

Donde, $n$ denota el número de capas, $\rho_{i}$ y $h_{i}$ son la resistividad y el espesor de la iésima capa respectivamente. La ecuación 2.4 es usada para calcular un modelo de respuesta en un SEV en corriente continua (DC).

De acuerdo a Ekinci \& Demirci (2008), la ecuación 2.5 proporciona una solución con mínimos cuadrados al problema de inversión de datos para la resistividad aparente. Para una inversión automática, frecuentemente se ajusta un valor inicial de los mismos datos observados para tener una convergencia inicial, y a medida que las iteraciones avancen capa por capa el error (ecuación 2.6) disminuye encontrando una solución estable para cada modelo analizado.

$$
\Delta p=\operatorname{Vdiag}\left\{\frac{\lambda_{i}}{\lambda^{2}{ }_{j}+\varepsilon^{2}}\right\} U^{T} \Delta d
$$

Donde,

$$
\varepsilon=\lambda_{L} \Delta X^{\frac{1}{L}}
$$

Finalmente, con base en los datos obtenidos de los sondeos verticales se realizó un modelo de predicción en 3D usando las propiedades de resistividad definidas en los SEV de la Fm. Colorado. El modelo fue realizado en el software libre Octave, el cual es una modificación del script propuesto por Ekinci \& Demirci (2008). Los resultados espaciales obtenidos en el software Octave para la resistividad eléctrica pueden ser organizados en 
forma matricial y visualizarse en 3D. Este proceso consta de una interpolación multivariable (x, y, z, resistividad). De forma algebraica la interpolación se realiza por medio de matrices multidimensionales (hipermatrices) que permiten distribuir una matriz 2D en una dimensión adicional. La gráfica obtenida permite la rotación y cortes (slides) en diferentes ejes, destacando así anisotropía o lineamientos en la resistividad del subsuelo. En la figura 7 se muestra la interpolación 3D para datos de resistividad menor a $10 \mathrm{ohm}-\mathrm{m}$. Este resultado matemático permite validar el método de procesamiento individual para los sondeos verticales, ya que se considera un espacio continuo entre las variables dependientes e independientes, por lo tanto, cada sondeo debe tener cierta correspondencia con otros que se encuentren a una distancia determinada.

\section{RESULTADOS}

TABLA II. VALORES TÍPICOS DE RESISTENCIA PARA VARIOS MATERIALES (ADVANCED GEOSCIENCES INCORPORATED, 2008; GIBSON AND GEORGE, 2003; LOKE \& LANE, 2004; SOCIETY OF EXPLORATION GEOPHYSICIST OF JAPAN, 2004; UNITED STATES CORPS OF ENGINEERS, 2001).

\begin{tabular}{|c|c|c|c|c|c|}
\hline \multirow{2}{*}{ Tipo de material } & \multicolumn{5}{c|}{ UNITED STATES CORPS OF ENGINEERS, 2001). } \\
\cline { 2 - 6 } & SEJ & USOCE & Gilbson & Loke - Lane & AGI \\
\hline Arcilla & $1-300$ & $1-20$ & $1-100$ & $1-100$ & $10-100$ \\
\hline Arena & $10-1.100$ & $20-100$ & $50-1.050$ & $10-800$ & $600-10.000$ \\
\hline Grava & $20-7.000$ & ---- & $100-1.400$ & $600-10.000$ & $600-10.000$ \\
\hline Caliza & --- & $100-1 \times 10^{6}$ & $50-1 \times 10^{6}$ & $50-400$ & $100-1 \times 10^{6}$ \\
\hline Shale & $2-200$ & $1-500$ & ---- & $20-2.000$ & ---- \\
\hline Arenisca & $10-700$ & ---- & $1-7.4 \times 10^{6}$ & $8-4000$ & $100-1000$ \\
\hline Granito & $300-40.000$ & ---- & $102-1 \times 10^{6}$ & $5.000-1 \times 10^{6}$ & ---- \\
\hline
\end{tabular}

\subsection{RESISTIVIDAD EN EL SUBSUELO}

Las condiciones naturales que afectan la resistividad eléctrica están directamente relacionadas con el flujo de corriente de los materiales. El flujo de corriente eléctrica es análogo al flujo del agua que se mueve siempre en dirección de la menor resistencia (Greenhouse \& Gudjurgis, 1998). La matriz para suelo y roca siempre está compuesta por una sustancia solido - poroso, la cual puede estar rellena de un fluido (agua o aire). Por lo tanto, para cada tipo de material existe un rango característico de resistividad. En la Tabla 2 se documenta la medida de resistividad para varios tipos de materiales.

Los perfiles invertidos de los SEVs muestran diferente número de capas, las cuales dependen únicamente del número de datos adquiridos en campo. La Tabla 3 muestra los resultados para el SEV-1, SEV-4 y SEV-7, los cuales se encuentran a una distancia superior a $1 \mathrm{~km}$ y abarcan una región representativa del Campo Colorado. 
TABLA III. RESULTADOS DE LOS PARÁMETROS A PARTIR DE LA INVERSIÓN DE LA RESISTIVIDAD APARENTE USANDO MÍNIMOS CUADRADOS PARA LOS TRES SONDEOS ELÉCTRICOS VERTICALES MÁS REPRESENTATIVOS POR SU LOCALIZACIÓN SOBRE EL CAMPO COLORADO

\begin{tabular}{|ll|ll|ll|}
\hline \multicolumn{2}{|c|}{ SEV-1 } & \multicolumn{2}{c|}{ SEV-4 } & \multicolumn{2}{c|}{ SEV-7 } \\
\hline \multicolumn{1}{|c|}{ metros } & \multicolumn{1}{|c|}{ m } & \multicolumn{1}{c|}{ metros } & \multicolumn{1}{c|}{ m } & metros \\
\hline$\rho_{1}=7.85$ & $h_{1}=0.51$ & $\rho_{1}=22.33$ & $h_{1}=0.53$ & $\rho_{1}=8.53$ & $h_{1}=1.07$ \\
$\rho_{2}=7.11$ & $h_{2}=3.16$ & $\rho_{2}=66.39$ & $h_{2}=0.92$ & $\rho_{2}=18.25$ & $h_{2}=2.79$ \\
$\rho_{3}=1.52$ & $h_{3}=4.80$ & $\rho_{3}=21.64$ & $h_{3}=1.62$ & $\rho_{3}=5.74$ & $h_{3}=5.05$ \\
$\rho_{4}=9.18$ & $h_{4}=5.98$ & $\rho_{4}=0.67$ & $h_{4}=3.14$ & $\rho_{4}=41.70$ & $h_{4}=9.57$ \\
$\rho_{5}=61.75$ & $h_{5}=10.81$ & $\rho_{5}=5.70$ & $h_{5}=4.11$ & $\rho_{5}=2.87$ & $h_{5}=14.57$ \\
$\rho_{6}=4.47$ & $h_{6}=15.81$ & $\rho_{6}=31.13$ & $h_{6}=5.53$ & & \\
& & $\rho_{7}=203.64$ & $h_{7}=10.94$ & & \\
& & $\rho_{8}=70.58$ & $h_{8}=13.41$ & & \\
\hline
\end{tabular}
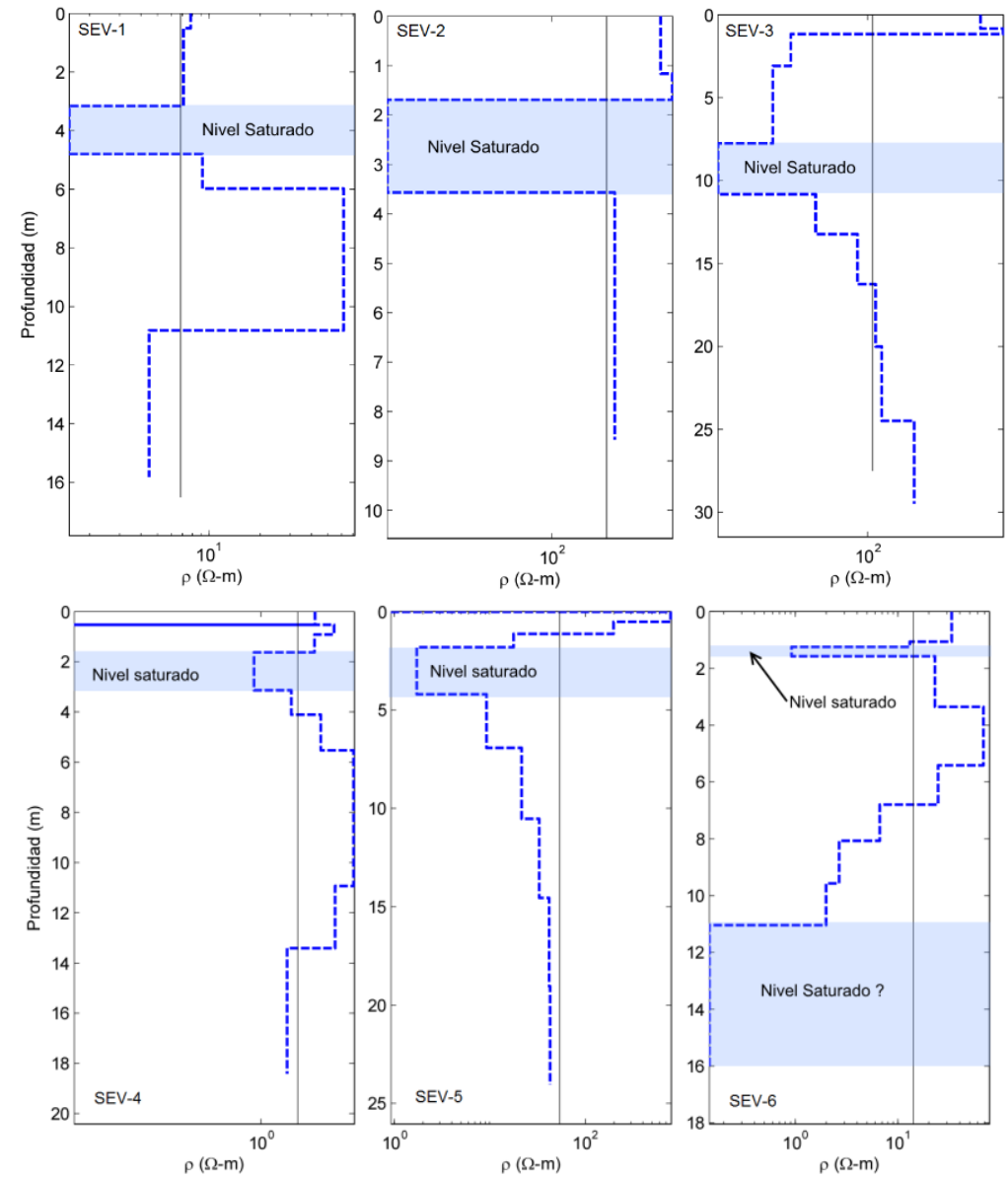

Figura 5. Perfiles de resistividad eléctrica para los SEV-1 al SEV-6 mostrando la distribución de 2 zonas saturadas, la primera con un espesor de $1-2.5 \mathrm{~m}$ y con mayor concentración a los $1.5 \mathrm{~m}$ de profundidad. La segunda zona con espesor de $6 \mathrm{~m}$ de espesor y con mayor concentración a los $15 \mathrm{~m}$ de profundidad. 

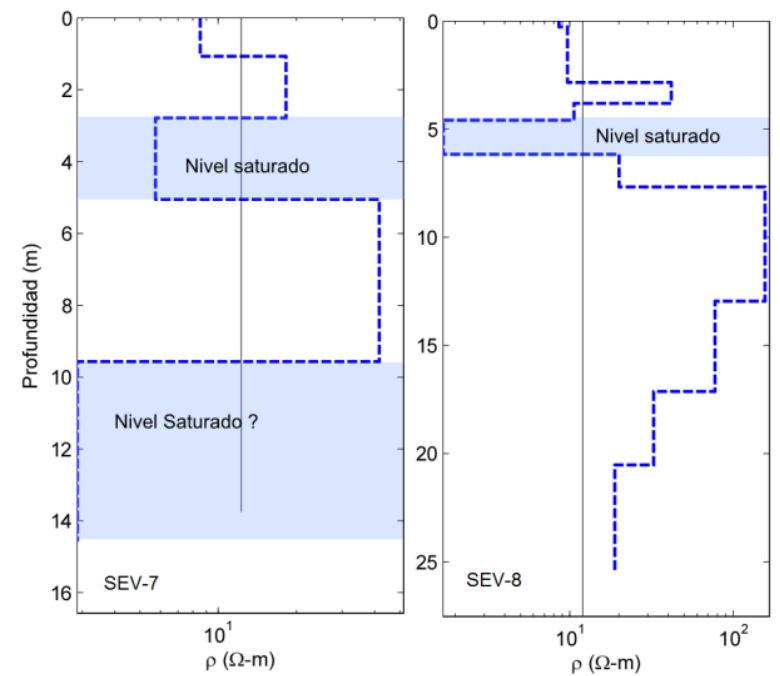

Figura 6. Perfiles de resistividad eléctrica para los SEV-7 y SEV-8 mostrando la distribución de 2 zonas saturadas, la primera con un espesor de $2 \mathrm{~m}$ y con mayor concentración a los $3 \mathrm{~m}$ de profundidadLa segunda zona con espesor de $4 \mathrm{~m}$ de espesor y con mayor concentración a los $15 \mathrm{~m}$ de profundidad.

Los valores de resistividad menores a 15 ohm-m corresponden a niveles de arenas saturadas como se observa en la Figura 5, con espesores entre 4 y 6 m. Los topes de esta capa de arenas saturadas se encuentran a 3 $\mathrm{m}$ y $10 \mathrm{~m}$ de profundidad, variando entre desde $3-7 \mathrm{~m}$ y entre los 10 - $14 \mathrm{~m}$, siendo la segunda la de mayor interés hidrogeológico debido a su espesor (Figuras 5 y 6). La delimitación de los niveles saturados en cada perfil permite estimar un rango típico de $1-10 \mathrm{ohm}-\mathrm{m}$, las profundidades varían entre 3 y $6 \mathrm{~m}$ para los SEV-1 hasta SEV-6 (Figura 5), siendo este resultado influenciado por el Caño El Caneo. En los SEV-6 y SEV-7 (Figuras 5 y 6) se estima un posible acuífero con profundidades entre los
11 y $16 \mathrm{~m}$, la localización de estos sondeos corresponde a la influencia del Rio La Colorada. Estos niveles saturados corresponden a suelos residuales arenosos y arcillosos de la Formación Colorado.

\subsection{MODELO 3D}

El modelo 3D permite sugerir que la continuidad lateral de las zonas saturadas se ve favorecida en dirección $\mathrm{N}-\mathrm{S}$, desarrollando capas acuíferas tabulares, es decir, un nivel piezómetro sub-horizontal, ideal para la extracción de agua con pozos. Esta zona saturada corresponde a una erosión muy leve de la Formación Colorado que genera suelos areno - arcillosos cuyo potencial como acuífero es muy alto y corresponden específicamente a las tonalidades azules (Figuras 7 y 8).

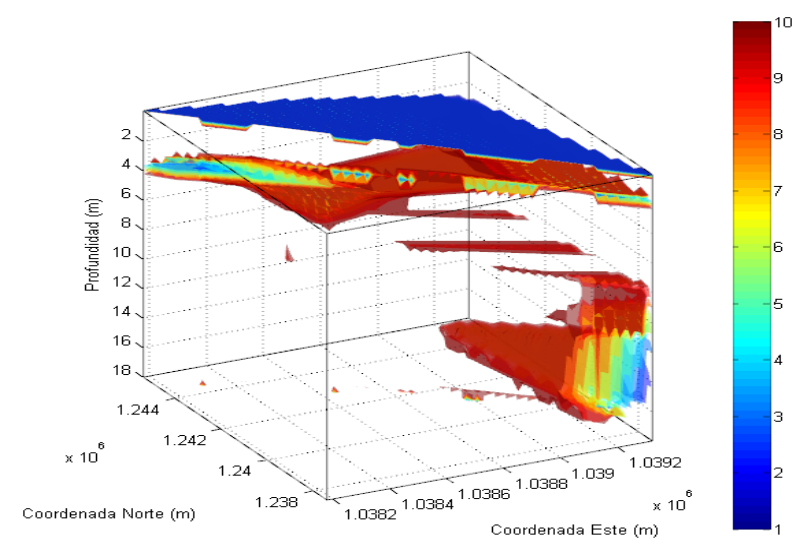

Figura 6. Modelo 3D de la zona de estudio mostrando la relación entre profundidad y resistividad. Los valores de resistividad para el modelo varían entre $1-10 \mathrm{ohm}-\mathrm{m}$, permitiendo observar la zona saturada y su distribución N-S y E-W.
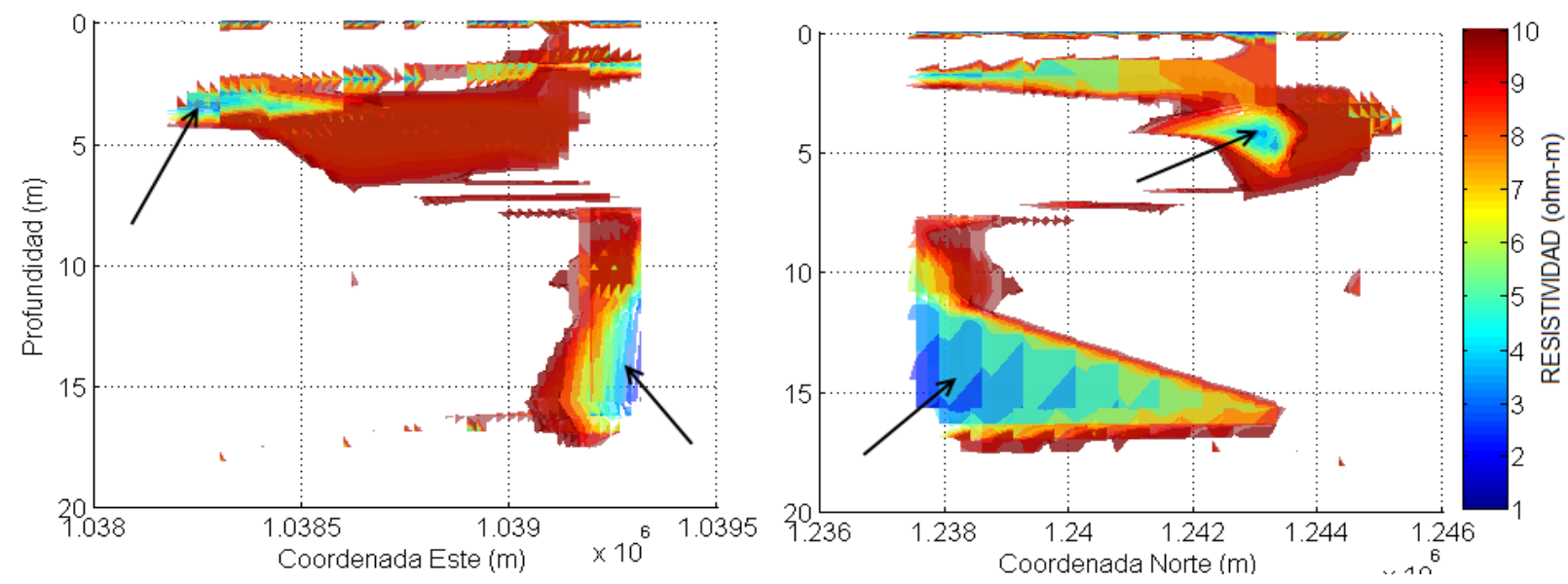

Figura 7. Secciones de resistividad eléctrica de rango 1 - 10 ohm-m en dirección E-W (izquierda) y en dirección N-S (derecha). Las flechas indican las zonas de más baja resistividad en color azul. 


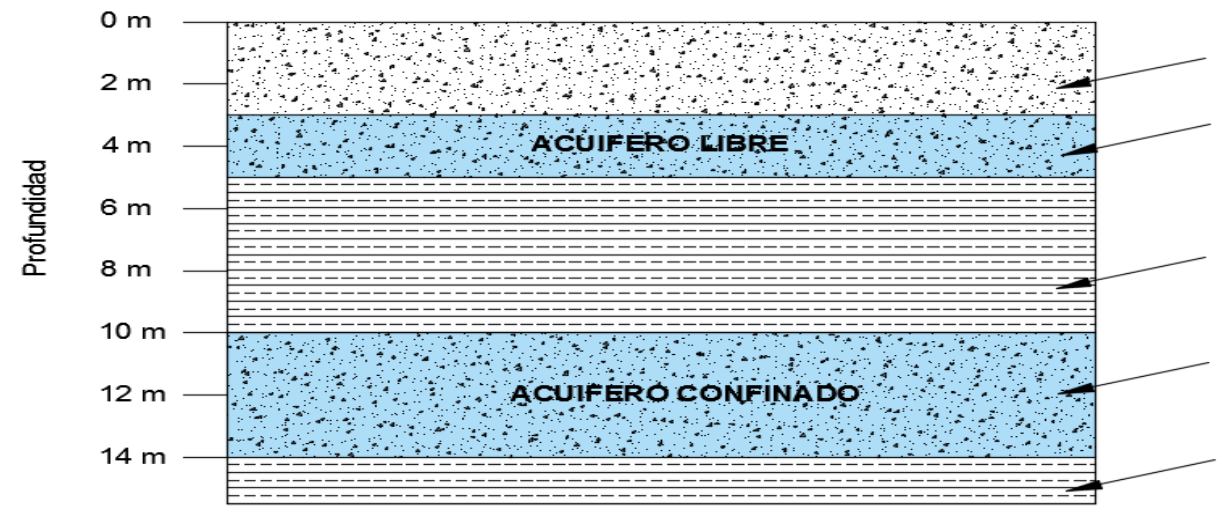

Suelo arenoso producto de la erosión de la Fm. Colorado

Primera zona acuifera de en arenas. Espesor de 2 metros. Discontinua lateralmente

Suelo Arcilloso producto de la erosión de la Fm. Colorado

Segunda zona acuifera de en arenas. Espesor de 4-6 metro: Continua lateralmente.

Suelo Arcilloso producto de la erosión de la Fm. Colorado

Figura 8. Modelo del subsuelo relacionando la estratigrafía y la resistividad, se resaltan las capas acuíferas y su potencial como acuífero libre y acuífero confinado

\section{CONCLUSIONES Y RECOMENDACIONES}

El procesamiento de los sondeos eléctricos verticales usando el método de mínimos cuadrados como parte del proceso de inversión de resistividades aparentes proporciona una interpretación acertada al asignar errores globales a cada capa. Por esto, el número de capas depende únicamente del número de datos que se adquieran en campo. Los ajustes entre las curvas de resistividad aparente de campo y teóricas pueden ser optimizadas utilizando otros algoritmos, como, por ejemplo, smooth line. Esto permite cambios detallados en el valor de resistividad invertida que se asigna a cada capa.

El potencial hidrogeológico para el campo Escuela Colorado se estima en un rango de profundidades entre los 2-6 m y $8-10 \mathrm{~m}$ (Figura 7) correspondientes a las zonas saturadas 1 y 2 respectivamente; asociados a suelos residuales y rocas fracturadas de la Formación Colorado (Toc). Según las resistividades de los perfiles en profundidad y de acuerdo al rango de valores propuestos en la Tabla 2 se propone un modelo del

subsuelo como un sistema de acuífero libre y acuífero confinado (Figura 8) siguiendo los planteamientos de Holzbecher (2007).

Para la zona estudiada es importante reconocer las características hidrogeológicas que permitan el prediseño de un sistema de agua potable a través de un pozo de bombeo somero. En este trabajo se muestra la posibilidad de realizar una exploración preliminar con continuidad lateral a costos bajos que permiten la explotación del agua subterránea con fines domiciliarios.

Para estimar una continuidad lateral más detallada se recomienda usar el método geofísico de Tomografía de Resistividad Eléctrica (TRE) y una perforación superficial para caracterizar la conductividad y permeabilidad de los materiales con el fin de simular un ensayo de bombeo y calcular el radio de influencia para diferentes zonas de las capas acuíferas halladas en este trabajo.

AGRADECIMIENTOS: El presente trabajo hace parte del Proyecto Modelo Geológico de Campo Escuela Colorado, los autores agradecemos a Campo Escuela Colorado y a la Vicerrectoría de Investigación y Extensión (VIE) de la UIS por el apoyo económico, administrativo y social para llevar a cabo esta y otras investigaciones relacionadas con el proyecto. También agradecemos a la continua colaboración de la escuela de Geología.

\section{REFERENCIAS}

[1] Advanced Geosciences Incorporated. 2009. Advanced Resistivity Imaging Seminar. Austin (Texas), April 16. 25p.

[2] Butler D. K. 2008. "What Is Near-Surface Geophysics." In Near-Surface Geophysics, by Dwain K. Butler, 1-6. Tulsa: Society of Exploration Geophysicist [3] Caballero V., Parra M., Mora Bohorquez R. 2010. Levantamiento de la cordillera oriental de Colombia durante el eoceno tardío - oligoceno temprano: proveniencia sedimentaria en el sinclinal de nuevo mundo, cuenca valle medio de la magdalena. Boletín de Geología 32 (1): 45-77.

[4] Ekinci Y. L. \& Demirci A. 2009. A Damped leastsquares inversion program for the interpretation of schlumberger sounding curves. Journal of Applied Sciences 8 (22): 4070-4078.

[5] Gómez E., Jordan T., Allmendnger R, Hegarty K., Kelley S. Syntectonic Cenozoic Sedimentation in the Northern Middle Magdalena Valley Basin of Colombia and Implications for Exhumation of the Northern Andes. Geological Society of America Bulletin, 547-569, 2008.

[6] Gibson P. J., \& George D. M., 2003. Environmental Applications of Geophysical Surveying Techniques. Hauppauge: Nova Science Publishers. 
[7] Greenhouse J. P. \& Gudjurgis D. S. 1998. Introduction to Environmental and Engineering Geophysics." SAGEEP (98). Chicago.

[8] Holzbecher, L. 2007. Environmental modeling using Matlab. Springer-Verlag, Berlin Heidelberg, 393p. LIBRO

[9] Hubbard J. L. 2009. Use of electrical resistivity and multichannel analysis of surface wave geophysical tomography in geotechnical site characterization of dam. Master of Science thesis. The University of Texas at Arlington.

[10] Koefoed, O. 1970. A fast method for determining the layer distribution from a raised kernel function in geoelectrical sounding. Geophysics prospect (18): 564570.

[11] Kearey P., Brooks M., and Hill I., 2002. An Introduction to geophysical exploration. Blackwell Science. 281p.

[12] Loke, M.H. \& Lane, J.W., 2004. Inversion of data from electrical resistivity imaging surveys in watercovered areas. Exploration Geophysics. (35) 266-271.

[13] Steeples D. 2001. Near-surface seismology: a short course. Lawrence: Society of Exploration Geophysics. [14] Society of Exploration Geophysicist of Japan. 2004. Application of Geophysical Methods to Engineering and Environmental Problems. Tokyo: Society of Exploration Geophysicist of Japan.

[15] United States Corps of Engineers. 2001. Geotechnical Investigations. Engineering Manual No. 1110-1-1804, Washington D.C.

[16] Zafra, M. 2013. Cartografía geológica del campo colorado a escala 1:25.000. Tesis de pregrado Universidad Industrial de Santander. 